\title{
Decay of Accelerated Protons and the Existence of the Fulling-Davies-Unruh Effect
}

\author{
Daniel A. T. Vanzella and George E. A. Matsas \\ Instituto de Física Teórica, Universidade Estadual Paulista, Rua Pamplona 145, 01405-900, São Paulo, São Paulo, Brazil
}

(Received 5 April 2001; published 25 September 2001)

\begin{abstract}
We investigate the weak decay of uniformly accelerated protons in the context of standard quantum field theory. Because the mean proper lifetime of a particle is a scalar, the same value for this observable must be obtained in the inertial and coaccelerated frames. We are only able to achieve this equality by considering the Fulling-Davies-Unruh effect. This reflects the fact that the Fulling-Davies-Unruh effect is mandatory for the consistency of quantum field theory.
\end{abstract}

DOI: $10.1103 /$ PhysRevLett.87.151301

A couple of years after the discovery by Hawking that black holes should evaporate [1], Unruh realized that many features present in the Hawking effect could be better understood in the simpler context of Minkowski spacetime [2]. As an extra bonus, he found that the Minkowski vacuum, i.e., the quantum state associated with the nonexistence of particles according to inertial observers, corresponds to a thermal bath of elementary particles at temperature $T_{\mathrm{FDU}}=a \hbar / 2 \pi k c$ as measured by uniformly accelerated observers with proper acceleration $a$. Indeed this reflects the fact that the particle content of a quantum field theory (QFT) is observer dependent, as noted by Fulling [3] and Davies [4] some time before. Thus while inertial observers in the Minkowski vacuum would be frozen at $0 \mathrm{~K}$, accelerated ones would be burned provided that their proper acceleration were high enough.

Perhaps partly because of its "paradoxical looking" and partly because of the technicalities involved in its derivation (see, e.g., Ref. [5]), the Fulling-Davies-Unruh (FDU) effect is still a source of much skepticism. As a consequence, much effort has been spent to devise ways of observing it (see, e.g., Ref. [6] and references therein for a comprehensive list). Since $T_{\mathrm{FDU}}=\left[a /\left(2.5 \times 10^{22} \mathrm{~cm} / \mathrm{s}^{2}\right)\right] \mathrm{K}$, direct manifestations of the FDU effect would be expected only under extremely high acceleration regimes. Very recently, e.g., Chen and Tajima suggested the possibility of observing the FDU effect by means of petawatt-class lasers with which $e^{-}$'s would reach accelerations of $\sim 10^{28} \mathrm{~cm} / \mathrm{s}^{2}$ in every laser cycle [7]. It is well known that accelerated $e^{-}$, s suffer recoil because of the radiation reaction force associated with the Larmor radiation. For instance, an $e^{-}$in a constant electric field $\mathbf{E}$ should quiver around a uniformly accelerated world line with proper acceleration $a=e|\mathbf{E}| / m_{e}$, where $e$ and $m_{e}$ are the electron charge and mass, respectively. Rather than using the radiation reaction force to calculate the $e^{-}$recoil, Chen and Tajima have estimated it by assuming that the quivering is a consequence of the random absorption of quanta from the FDU thermal bath as seen in the $e^{-}$, s proper frame. Inspired by this, they call the recoil-induced photon emission "Unruh radiation." Eventually they calculate the emitted power associated with the Unruh radiation
PACS numbers: 04.62.+v, 13.30.Ce, 23.40.Bw

for an $e^{-}$during each laser half cycle and argue that its observation would consist of an experimental test for the FDU effect.

Here we look at this issue from a distinct point of view. Rather than looking for an experimental manifestation of the FDU effect when high accelerations are achieved, which, in general, leads to paramount technical problems [8], we will take a theoretic-oriented strategy. This sort of approach is not new [9-11] but we hope that the comprehensive understanding brought by the FDU effect to the decay of accelerated $p^{+}$'s (which is a potentially important phenomenon in its own right [12]) will be very convincing of the necessity of this effect for the consistency of QFT. First, we will analyze in the inertial frame and using standard QFT the decay of uniformly accelerated $p^{+}$'s and next we will show that the FDU effect is essential to reproduce the proper decay rate in the uniformly accelerated frame.

According to the standard model, inertial $p^{+}$'s are stable, which is in agreement with highly accurate experiments $\left(\tau_{p}>1.6 \times 10^{25} \mathrm{yr}\right)$ [13]. As far as we know, the first ones to comment that noninertial $p^{+}$'s could decay were Ginzburg and Syrovatskii [14] but no calculations were performed until Muller [15] obtained an estimation of the decay rate associated with the process

$$
\text { (i) } \quad p^{+} \stackrel{a}{\rightarrow} n^{0} e^{+} \nu_{e}
$$

by assuming that all the involved particles are scalars. A more realistic calculation describing the leptons as fermions was only performed very recently by the authors [16]. The energy scale of the emitted particles in the $p^{+}$instantaneous inertial rest frame is of the order of the $p^{+}$proper acceleration $a$. Thus if $a \ll m_{Z^{0}}, m_{W^{ \pm}}\left(\approx 10^{36} \mathrm{~cm} / \mathrm{s}^{2}\right)$, a Fermi-like effective theory can be used. The effective coupling constant is fixed such that the $\beta$-decay rate for inertial $n^{0}$ 's be compatible with observation, i.e., leads to a mean proper lifetime of $887 \mathrm{~s}$ [13].

For our present purposes it is enough to analyze reaction (i) in a 2-dimensional spacetime. Hereafter we use signature (+-) and natural units $k_{B}=c=\hbar=1$ unless stated otherwise. The world line of a uniformly accelerated $p^{+}$in usual Cartesian coordinates of Minkowski spacetime is given by $z^{2}-t^{2}=a^{-1}$ where $\sqrt{a^{\mu} a_{\mu}}=a=$ const is 
the $p^{+}$proper acceleration. We construct, thus, the vector current $j^{\mu}=q u^{\mu} \delta\left(\sqrt{z^{2}-t^{2}}-a^{-1}\right)$ associated with a uniformly accelerated classical $p^{+}$with 4 -velocity $u^{\mu}$, where $q$, at this point, is an arbitrary parameter.

In order to allow the $p^{+}$to decay, we shall endow the current with an internal degree of freedom. For this purpose we shall promote $q$ to a self-adjoint operator $\hat{q}(\tau)$ $[17,18]$ acting on a 2-dimensional Hilbert space associated with proton $|p\rangle$ and neutron $|n\rangle$ states. They will be assumed to be energy eigenstates of the proper free Hamiltonian $\hat{H}$ of the proton/neutron system: $\hat{H}|p\rangle=m_{p}|p\rangle$, $\hat{H}|n\rangle=m_{n}|n\rangle$, where $m_{p}$ and $m_{n}$ are the $p^{+}$and $n^{0}$ masses, respectively. In this context, $|p\rangle$ and $|n\rangle$ will be seen as unexcited and excited states of the nucleon, respectively. Further we will define the effective Fermi constant as $G_{F} \equiv|\langle p|\hat{q}(0)| n\rangle|$, where $\hat{q}(\tau) \equiv e^{i \hat{H} \tau} \hat{q}(0) e^{-i \hat{H} \tau}$ and $\tau$ is the $p^{+}$proper time.

In the inertial frame, the fermionic fields describing the leptons in (i) can be written as

$$
\hat{\Psi}(t, z)=\sum_{\sigma= \pm} \int_{-\infty}^{+\infty} d k\left(\hat{a}_{k \sigma} \psi_{k \sigma}^{(+\omega)}+\hat{c}_{k \sigma}^{\dagger} \psi_{-k-\sigma}^{(-\omega)}\right),
$$

where $\omega=\sqrt{m^{2}+k^{2}} \geq m$, and $m, k$, and $\sigma$ represent mass, momentum, and polarization quantum numbers, respectively. In the Dirac representation [19], the Minkowski modes, i.e., the ones defined with respect to the inertial Killing field $\partial / \partial t$, are $\psi_{k \sigma}^{( \pm \omega)}(t, z) \equiv$ $\lambda_{k \sigma}^{( \pm \omega)} e^{i(\mp \omega t+k z)} / \sqrt{2 \pi}$ with

$$
\lambda_{k+}^{( \pm \omega)}=\left(\begin{array}{c} 
\pm \sqrt{(\omega \pm m) / 2 \omega} \\
0 \\
k / \sqrt{2 \omega(\omega \pm m)} \\
0
\end{array}\right),
$$

and

$$
\lambda_{k-}^{( \pm \omega)}=\left(\begin{array}{c}
0 \\
\pm \sqrt{(\omega \pm m) / 2 \omega} \\
0 \\
-k / \sqrt{2 \omega(\omega \pm m)}
\end{array}\right) .
$$

Then the annihilation $\hat{a}_{k \sigma}, \hat{c}_{k \sigma}$ and creation $\hat{a}_{k \sigma}^{\dagger}, \hat{c}_{k \sigma}^{\dagger}$ operators satisfy $\left\{\hat{a}_{k \sigma}, \hat{a}_{k^{\prime} \sigma^{\prime}}^{\dagger}\right\}=\left\{\hat{c}_{k \sigma}, \hat{c}_{k^{\prime} \sigma^{\prime}}^{\dagger}\right\}=\delta\left(k-k^{\prime}\right) \delta_{\sigma \sigma^{\prime}}$ and $\left\{\hat{a}_{k \sigma}, \hat{a}_{k^{\prime} \sigma^{\prime}}\right\}=\left\{\hat{c}_{k \sigma}, \hat{c}_{k^{\prime} \sigma^{\prime}}\right\}=\left\{\hat{a}_{k \sigma}, \hat{c}_{k^{\prime} \sigma^{\prime}}\right\}=\left\{\hat{a}_{k \sigma}\right.$, $\left.\hat{c}_{k^{\prime} \sigma^{\prime}}^{\dagger}\right\}=0$.

Let us assume that the electron and neutrino fields are coupled to the nucleon current according to the Fermi-like action

$$
\hat{S}_{I}=\int d^{2} x \sqrt{-g} \hat{j}_{\mu}\left(\hat{\bar{\Psi}}_{\nu} \gamma^{\mu} \hat{\Psi}_{e}+\hat{\bar{\Psi}}_{e} \gamma^{\mu} \hat{\Psi}_{\nu}\right) .
$$

(The choice of other interaction actions would not change conceptually our final conclusions.)

The $p^{+}$proper decay rate is written, thus, as

$$
\Gamma_{(\mathrm{i})}^{p \rightarrow n}=\frac{1}{T} \sum_{\sigma_{e}, \sigma_{\nu}= \pm} \int_{-\infty}^{+\infty} d k_{e} \int_{-\infty}^{+\infty} d k_{\nu}\left|\mathcal{A}_{(\mathrm{i})}^{p \rightarrow n}\right|^{2},
$$

where $\mathcal{A}_{(\mathrm{i})}^{p \rightarrow n}=\left\langle n\left|\otimes\left\langle e_{k_{e} \sigma_{e}}^{+}, \nu_{k_{\nu} \sigma_{\nu}}\left|\hat{S}_{I}\right| 0\right\rangle \otimes\right| p\right\rangle$ is the decay amplitude, at the tree level, and $T$ is the $p^{+}$total proper time. Eventually, we obtain

$$
\begin{aligned}
\Gamma_{(\mathrm{i})}^{p \rightarrow n}= & \frac{G_{F}^{2} \tilde{m}_{e} a}{2 \pi^{3 / 2} e^{\pi \Delta m}} \\
& \times G_{13}^{30}\left(\left.\tilde{m}_{e}^{2}\right|_{-1 / 2,1 / 2+i \widetilde{\Delta m}, 1 / 2-i \widetilde{\Delta m})}\right),
\end{aligned}
$$

where $G_{p q}^{m n}$ is the Meijer function [20], $\Delta m \equiv m_{n}-m_{p}$, $\widetilde{\Delta m} \equiv \Delta m / a, \tilde{m}_{e} \equiv m_{e} / a$, and we have assumed $m_{\nu}=$ 0 . The value of the effective Fermi constant $G_{F}$ is fixed from phenomenology. By making $\Delta m \rightarrow-\Delta m$ and $a \rightarrow$ 0 in Eq. (5), we obtain that the mean proper lifetime of inertial $n^{0}$ 's due to $\beta$ decay,

(ii) $n^{0} \rightarrow p^{+} e^{-} \bar{\nu}_{e}$,

is $\tau_{\text {(ii) }}^{n \rightarrow p}=1 / \Gamma_{(\mathrm{ii})}^{n \rightarrow p}=\pi /\left(2 G_{F}^{2} \sqrt{\Delta m^{2}-m_{e}^{2}}\right)$. Now let us assume that the $n^{0}$ mean lifetime in 2 dimensions is, e.g., 887 s. In this case, we obtain $G_{F}=9.92 \times 10^{-13}$. Note that $G_{F} \ll 1$, which corroborates our perturbative approach. Now we are able to plot in Fig. 1 the $p^{+}$mean proper lifetime $\tau_{(\mathrm{i})}^{p \rightarrow n}=1 / \Gamma_{(\mathrm{i})}^{p \rightarrow n}$ [see Eq. (5)] as a function of $a$. (The necessary energy to allow $p^{+ \text {'s }}$ to decay is provided by the external accelerating agent.)

Now let us describe the $p^{+}$decay from the point of view of coaccelerated observers according to which the $p^{+}$is immersed in a FDU thermal bath at a temperature $T_{\mathrm{FDU}}=a / 2 \pi$. According to them, process (i) is forbidden from energy conservation (since the $p^{+}$is static) but the following ones

$$
\begin{gathered}
p^{+} e^{-} \stackrel{a}{\rightarrow} n^{0} \nu_{e}, \quad \text { (iv) } \quad p^{+} \bar{\nu}_{e} \stackrel{a}{\rightarrow} n^{0} e^{+}, \\
\text {(v) } \quad p^{+} e^{-} \bar{\nu}_{e} \stackrel{a}{\rightarrow} n^{0}
\end{gathered}
$$

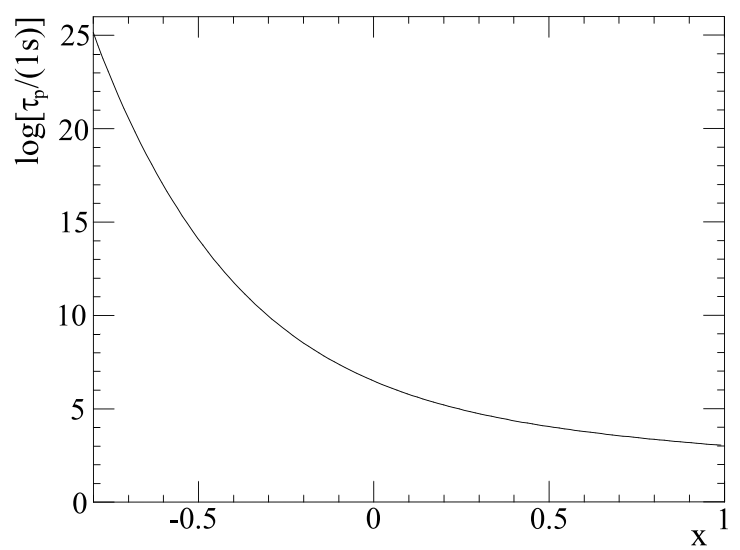

FIG. 1. $T_{p} \equiv \log _{10}\left[\tau_{p} /(1 \mathrm{~s})\right]$ is plotted as a function of $x \equiv \log _{10}[a /(1 \mathrm{MeV})]$, where $\tau_{p}$ is the $p^{+}$mean proper lifetime, $a$ its proper acceleration, $m_{e}=0.511 \mathrm{MeV}$, and $\Delta m=1.29 \mathrm{MeV} . \quad\left(1 \mathrm{MeV} \approx 4.6 \times 10^{31} \mathrm{~cm} / \mathrm{s}^{2}\right.$. $)$ Note that $\tau_{p} \rightarrow+\infty$ for inertial $p^{+}$'s. 
become allowed since the $p^{+}$can interact with the leptons of the thermal bath. By comparing process (i) against processes (iii)-(v), we can see how different are the descriptions given by the inertial and accelerated observers.

The suitable coordinates to analyze the $p^{+}$decay according to uniformly accelerated observers are the Rindler ones $(v, u)$. They are related with the usual Cartesian coordinates by $t=u \sinh v, z=u \cosh v$, where $0<u<$ $+\infty$ and $-\infty<v<+\infty$. In these coordinates, the line element of Minkowski spacetime at the Rindler wedge $(x>|t|)$ is $d s^{2}=u^{2} d v^{2}-d u^{2}$ and the world line of a $p^{+}$with proper acceleration $a$ is $u=a^{-1}=$ const.

According to uniformly accelerated observers, the fermionic field is expanded as [21]

$$
\hat{\Psi}(v, u)=\sum_{\sigma= \pm} \int_{0}^{+\infty} d \bar{\omega}\left(\hat{b}_{\bar{\omega} \sigma} \chi_{\bar{\omega} \sigma}+\hat{d}_{\bar{\omega} \sigma \chi-\bar{\omega}-\sigma}^{\dagger}\right),
$$

where we recall that Rindler frequencies $\bar{\omega}$ may assume arbitrary positive real values since they do not obey any dispersion relation. Here, $\chi_{\bar{\omega} \sigma}(v, u) \equiv C_{\bar{\omega}} \xi_{\bar{\omega} \sigma} e^{-i \bar{\omega} v / a}$ where $C_{\bar{\omega}} \equiv \sqrt{[m \cosh (\pi \bar{\omega} / a)] /\left[2 \pi^{2} a\right]}$ and

$$
\begin{gathered}
\xi_{\bar{\omega}+}=\left(\begin{array}{c}
K_{i \bar{\omega} / a+1 / 2}(m u)+i K_{i \bar{\omega} / a-1 / 2}(m u) \\
0 \\
-K_{i \bar{\omega} / a+1 / 2}(m u)+i K_{i \bar{\omega} / a-1 / 2}(m u) \\
0
\end{array}\right), \\
\xi_{\bar{\omega}-=}=\left(\begin{array}{c}
0 \\
K_{i \bar{\omega} / a+1 / 2}(m u)+i K_{i \bar{\omega} / a-1 / 2}(m u) \\
0 \\
K_{i \bar{\omega} / a+1 / 2}(m u)-i K_{i \bar{\omega} / a-1 / 2}(m u)
\end{array}\right)
\end{gathered}
$$

are positive and negative frequency Rindler modes, i.e., the ones defined with respect to the boost Killing field $a \partial / \partial v$. They are orthonormalized such that the annihilation $b_{\bar{\omega} \sigma}, d_{\bar{\omega} \sigma}$ and creation $b_{\bar{\omega} \sigma}^{\dagger}, d_{\bar{\omega} \sigma}^{\dagger}$ operators satisfy $\left\{\hat{b}_{\bar{\omega} \sigma}, \hat{b}_{\bar{\omega}^{\prime} \sigma^{\prime}}^{\dagger}\right\}=\left\{\hat{d}_{\bar{\omega} \sigma}, \hat{d}_{\bar{\omega}^{\prime} \sigma^{\prime}}^{\dagger}\right\}=\delta\left(\bar{\omega}-\bar{\omega}^{\prime}\right) \delta_{\sigma \sigma^{\prime}}$ and also $\left\{\hat{b}_{\bar{\omega} \sigma}, \hat{b}_{\bar{\omega}^{\prime} \sigma^{\prime}}\right\}=\left\{\hat{d}_{\bar{\omega} \sigma}, \hat{d}_{\bar{\omega}^{\prime} \sigma^{\prime}}\right\}=\left\{\hat{b}_{\bar{\omega} \sigma}, \hat{d}_{\bar{\omega}^{\prime} \sigma^{\prime}}\right\}=$ $\left\{\hat{b}_{\bar{\omega} \sigma}, \hat{d}_{\bar{\omega}^{\prime} \sigma^{\prime}}^{\dagger}\right\}=0$.

The transition rates associated with processes (iii) $-(\mathrm{v})$ are given by

$$
\begin{aligned}
\Gamma_{(\mathrm{iii})}^{p \rightarrow n}= & \frac{1}{T} \sum_{\sigma_{e^{-}}, \sigma_{\nu}= \pm} \int_{0}^{+\infty} d \bar{\omega}_{e^{-}} \int_{0}^{+\infty} d \bar{\omega}_{\nu}\left|\mathcal{A}_{(\mathrm{iii})}^{p \rightarrow n}\right|^{2} \\
& \times n_{F}\left(\bar{\omega}_{e^{-}}\right)\left[1-n_{F}\left(\bar{\omega}_{\nu}\right)\right] \\
\Gamma_{(\mathrm{iv})}^{p \rightarrow n}= & \frac{1}{T} \sum_{\sigma_{e^{+}}, \sigma_{\bar{\nu}}= \pm} \int_{0}^{+\infty} d \bar{\omega}_{e^{+}} \int_{0}^{+\infty} d \bar{\omega}_{\bar{\nu}}\left|\mathcal{A}_{(\mathrm{iv})}^{p \rightarrow n}\right|^{2} \\
& \times n_{F}\left(\bar{\omega}_{\bar{\nu}}\right)\left[1-n_{F}\left(\bar{\omega}_{e^{+}}\right)\right] \\
\Gamma_{(\mathrm{v})}^{p \rightarrow n}= & \frac{1}{T} \sum_{\sigma_{e^{-}}, \sigma_{\bar{\nu}}= \pm} \int_{0}^{+\infty} d \bar{\omega}_{e^{-}} \int_{0}^{+\infty} d \bar{\omega}_{\bar{\nu}}\left|\mathcal{A}_{(\mathrm{v})}^{p \rightarrow n}\right|^{2} \\
& \times n_{F}\left(\bar{\omega}_{e^{-}}\right) n_{F}\left(\bar{\omega}_{\bar{\nu}}\right),
\end{aligned}
$$

where at the tree level

$$
\begin{aligned}
& \mathcal{A}_{\text {(iii) }}^{p \rightarrow n}=\left\langle n\left|\otimes\left\langle\nu_{\bar{\omega}_{\nu} \sigma_{\nu}}\left|\hat{S}_{I}\right| e_{\bar{\omega}_{e^{-}} \sigma_{e^{-}}}^{-}\right\rangle \otimes\right| p\right\rangle, \\
& \mathcal{A}_{\text {(iv) }}^{p \rightarrow n}=\left\langle n\left|\otimes\left\langle e_{\bar{\omega}_{e^{+}} \sigma_{e^{+}}}\left|\hat{S}_{I}\right| \bar{\nu}_{\bar{\omega}_{\bar{\nu}} \sigma_{\bar{\nu}}}\right\rangle \otimes\right| p\right\rangle, \\
& \mathcal{A}_{(\mathrm{v})}^{p \rightarrow n}=\langle n| \otimes\left\langle 0\left|\hat{S}_{I}\right| e_{\bar{\omega}_{e^{-}} \sigma_{e^{-}}}^{\left.\bar{\nu}_{\bar{\omega}_{\bar{\nu}} \sigma_{\bar{\nu}}}\right\rangle \otimes|p\rangle,}\right.
\end{aligned}
$$

and we recall that in the Rindler wedge the $\gamma^{\mu}$ in $\hat{S}_{I}$ [see Eq. (4)] should be replaced by $\gamma_{R}^{\mu}$ (see Ref. [21]). Here $n_{F}(\bar{\omega}) \equiv 1 /\left(1+e^{\bar{\omega} / T_{\mathrm{FDU}}}\right)$ is the fermionic thermal factor which appears because of the presence of the FDU thermal bath.

After some calculations, we obtain

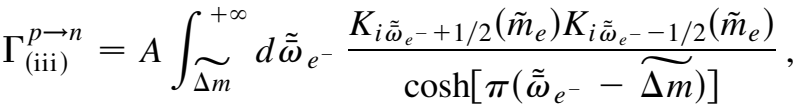

$$
\begin{aligned}
& \Gamma_{\text {(iv) }}^{p \rightarrow n}=A \int_{0}^{+\infty} d \tilde{\bar{\omega}}_{e^{+}} \frac{K_{i} \tilde{\tilde{\omega}}_{e^{+}+1 / 2}\left(\tilde{m}_{e}\right) K_{i} \tilde{\tilde{\omega}}_{e^{+}}-1 / 2}{\cosh \left[\pi\left(\tilde{\bar{m}}_{e}\right)\right.}, \\
& \Gamma_{(\mathrm{v})}^{p \rightarrow n}=A \int_{0}^{\widetilde{\Delta m}} d \tilde{\tilde{\omega}}_{e^{-}} \frac{K_{i} \tilde{\bar{\omega}}_{e^{-}}+1 / 2\left(\tilde{m}_{e}\right) K_{i} \tilde{\tilde{\omega}}_{e^{-}-1 / 2}\left(\tilde{m}_{e}\right)}{\cosh \left[\pi\left(\tilde{\tilde{\omega}}_{e^{-}}-\widetilde{\Delta m}\right)\right]},
\end{aligned}
$$

where $A \equiv\left(G_{F}^{2} \tilde{m}_{e} a\right) /\left(\pi^{2} e^{\pi \widetilde{\Delta m}}\right)$. A branching ratio analysis [22] indicates that for small accelerations, where "few" high-energy particles are available in the FDU thermal bath, process (v) dominates over processes (iii) and (iv), while for high accelerations, processes (iii) and (iv) dominate over process (v).

The $p^{+}$total proper decay rate is obtained by adding up all contributions:

$$
\begin{aligned}
\Gamma_{\mathrm{tot}}^{p \rightarrow n} & =\Gamma_{(\mathrm{iii})}^{p \rightarrow n}+\Gamma_{\text {(iv) }}^{p \rightarrow n}+\Gamma_{(\mathrm{v})}^{p \rightarrow n} \\
& =A \int_{-\infty}^{+\infty} d \tilde{\overline{\bar{\omega}}} \frac{K_{i \tilde{\bar{\omega}}+1 / 2}\left(\tilde{m}_{e}\right) K_{i \tilde{\bar{\omega}}-1 / 2}}{\cosh [\pi(\tilde{\bar{\omega}}-\widetilde{\Delta m})]} .
\end{aligned}
$$

Now, $\Gamma_{(\mathrm{i})}^{p \rightarrow n}$ and $\Gamma_{\text {tot }}^{p \rightarrow n}$ must coincide. Equation (9) is difficult to solve analytically because the integral variable is in the function index. (This can be seen as reflecting the essentially distinct inertial and coaccelerated frame calculations.) Hence we solve Eq. (9) numerically. Finally, by plotting $\tau_{\text {tot }}=1 / \Gamma_{\text {tot }}^{p \rightarrow n}$ as a function of $a$, we precisely obtain Fig. 1 [23]. We emphasize that we would not have obtained any agreement if we did not assume the FDU effect.

The confusion about what the FDU effect means has led to a number of erroneous conclusions in the literature. For instance, a $p^{+}$with proper acceleration $a=$ const in the Minkowski vacuum does not have to behave as if it were static in a (usual) Minkowski thermal bath at a temperature $T=a / 2 \pi$. (The FDU effect does not ensure any such coincidence.) The FDU effect can be rigorously derived [24] from the general Bisognano and Wichmann's theorem [25] obtained independently from axiomatic QFT (which is not even restricted to linear quantum fields). Moreover the necessity of the FDU effect for the consistency of the (successfully tested) standard QFT in Minkowski spacetime means that this effect was already observed [26]. We have illustrated it through the decay of accelerated $p^{+}$'s but other situations can be devised. Concerning 
electromagnetic processes, e.g., the FDU thermal bath is crucial to reproduce the response of a uniformly accelerated $e^{-}$to the Larmor radiation in the coaccelerated frame [10]. The same must be true if one takes into account the extra radiation induced by the $e^{-}$recoil. There is no question about the existence of the FDU effect provided one accepts the validity of the results obtained with standard QFT in flat spacetime.

G. M. is deeply indebted to A. Higuchi, D. Sudarsky, and R. Wald for discussions on QFT in noninertial frames since long time. The authors would like to acknowledge particularly A. Higuchi for discussions at early stages of this work. G. M. and D. V. were supported by Conselho Nacional de Desenvolvimento Científico e Tecnológico (partially) and Fundação de Amparo à Pesquisa do Estado de São Paulo (fully), respectively.

[1] S. W. Hawking, Nature (London) 248, 30 (1974); Commun. Math. Phys. 43, 199 (1975).

[2] W. G. Unruh, Phys. Rev. D 14, 870 (1976).

[3] S. A. Fulling, Phys. Rev. D 7, 2850 (1973).

[4] P. C. W. Davies, J. Phys. A 8, 609 (1975).

[5] R. M. Wald, Quantum Field Theory in Curved Spacetime and Black Hole Thermodynamics, Chicago Lectures in Physics (University of Chicago, Chicago, 1994).

[6] M. Visser, in “Experimental Unruh Radiation?", edited by J. Pullin, Newsletter of the Topical Group on Gravitation of the APS, gr-qc/0102044; H. Rosu, Gravitation Cosmol. 7, 1 (2001).

[7] P. Chen and T. Tajima, Phys. Rev. Lett. 83, 256 (1999).

[8] J. M. Leinaas, in Proceedings of the 18th Advanced ICFA, Beam Dynamics Workshop on Quantum Aspects of Beam Physics (hep-th/0101054).

[9] W. Unruh and R. M. Wald, Phys. Rev. D 29, 1047 (1984).
[10] A. Higuchi, G. E. A. Matsas, and D. Sudarsky, Phys. Rev. D 45, R3308 (1992); 46, 3450 (1992).

[11] G.E. A. Matsas, Phys. Lett. B 380, 24 (1996).

[12] A. Tokuhisa and T. Kajino, Astrophys. J. 525, L117 (1999).

[13] Particle Data Group, C. Caso et al., Eur. Phys. J. C 3, 1 (1998).

[14] V. L. Ginzburg and S. I. Syrovatskii, Usp. Fiz. Nauk 87, 65 (1965) [Annu. Rev. Astron. Astrophys. 3, 297 (1965)].

[15] R. Muller, Phys. Rev. D 56, 953 (1997).

[16] D. A.T. Vanzella and G.E. A. Matsas, Phys. Rev. D 63, 014010 (2001).

[17] B. S. DeWitt, General Relativity, edited by S. W. Hawking and W. Israel (Cambridge University Press, Cambridge, 1979).

[18] N.D. Birrell and P.C. W. Davies, Quantum Field Theory in Curved Space (Cambridge University Press, Cambridge, 1982).

[19] C. Itzykson and J.B. Zuber, Quantum Field Theory (McGraw-Hill, New York, 1980).

[20] I. S. Gradshteyn and I. M. Ryzhik, Table of Integrals, Series and Products (Academic Press, New York, 1980).

[21] P. Candelas and D. Deutsch, Proc. R. Soc. London A 362, 251 (1978); M. Soffel, B. Muller, and W. Greiner, Phys. Rev. D 22, 1935 (1980); R. Járegui, M. Torres, and S. Hacyan, Phys. Rev. D 43, 3979 (1991); E. Bautista, Phys. Rev. D 48, 783 (1993).

[22] G.E. A. Matsas and D. A. T. Vanzella, Phys. Rev. D 59, 094004 (1999).

[23] Indeed, we get $\Delta^{-1} \int_{\Delta} d x\left[\left(\tau_{\text {tot }}-\tau_{(\mathrm{i})}\right) / \tau_{\text {tot }}\right]^{2} \sim 10^{-16}$, where $\Delta$ is the integration interval used in Fig. 1.

[24] G. L. Sewell, Ann. Phys. (N.Y.) 141, 201 (1982).

[25] J. J. Bisognano and E. H. Wichmann, J. Math. Phys. (N.Y.) 17, 303 (1976).

[26] This is analogous to saying that centrifugal and Coriolis forces must exist just because otherwise noninertial observers would not be able to reproduce the observables calculated by inertial ones. 